\title{
Effect of ferrous/ferric ions molar ratio on reaction mechanism for hydrothermal synthesis of magnetite nanoparticles
}

\author{
N MIZUTANI, T IWASAKI*, S WATANO, T YANAGIDA ${ }^{\dagger}$, H TANAKA ${ }^{\dagger}$ and T KAWAI ${ }^{\dagger}$ \\ Department of Chemical Engineering, Osaka Prefecture University, 1-1 Gakuen-cho, Nakaku, Sakai, \\ Osaka 599-8531, Japan \\ ${ }^{\dagger}$ Institute of Scientific and Industrial Research, Osaka University, 8-1 Mihogaoka, Ibaraki, Osaka 567-0047, Japan
}

MS received 25 November 2007; revised 8 January 2008

\begin{abstract}
Magnetite nanoparticles were prepared by hydrothermal synthesis under various initial ferrous/ferric molar ratios without adding any oxidizing and reducing agents in order to clarify effects of the molar ratio on the reaction mechanism for the formation of magnetite nanoparticles. The magnetite nanoparticles prepared were characterized by a scanning electron microscope, powder X-ray diffractometer, and superconducting quantum interference device (SQUID). At the molar ratio corresponding to the stoichiometric ratio in the synthesis reaction of magnetite from ferrous hydroxide and goethite, the nucleation of magnetite crystals progressed rapidly in an initial stage of the hydrothermal synthesis, resulting in formation of the magnetite nanoparticles having a smaller size and a lower crystallinity. On the other hand, at higher molar ratios, the particle size and crystallinity increased with increasing molar ratio because using surplus ferrous hydroxide the crystallites of magnetite nanoparticles grew up slowly under hydrothermal conditions according to the Schikorr reaction. The magnetite nanoparticles prepared under various molar ratios had good magnetic properties regardless of the molar ratio.
\end{abstract}

Keywords. $\mathrm{Fe}_{3} \mathrm{O}_{4}$ nanoparticles; particle size; crystallinity; magnetic property; Schikorr reaction.

\section{Introduction}

Magnetite $\left(\mathrm{Fe}_{3} \mathrm{O}_{4}\right)$ is an important member of spinel type ferrite, and fine magnetite particles are widely used for recording materials, pigments, electrophotographic developers, etc (Fan et al 2001). Recently, as the applications expand, the demand for magnetite nanoparticles increases. Various methods for preparing magnetite nanoparticles have been developed. Liquid phase methods, for example, micro-emulsion (Zhou et al 2001; Gotić et al 2007), thermal decomposition of iron complex in an organic solvent (Park et al 2004), and co-precipitation from the $\mathrm{Fe}^{2+} /$ $\mathrm{Fe}^{3+}$-mixed salt solution in an alkaline medium (Wang et al 2007), are useful among them because they can prepare magnetite nanoparticles quite easily with homogeneous properties. However, the liquid phase methods have some problems. The magnetite nanoparticles prepared by the methods have relatively low crystallinity and organic solvents used in the preparation should be desired to be removed from the suspension containing the magnetite nanoparticles.

As an effective method to solve the problems, hydrothermal methods without using any organic solvent have attracted attention. For example, Qian et al (1994) re-

*Author for correspondence (iwasaki@chemeng.osakafu-u.ac.jp) ported the hydrothermal approach to prepare ultra fine magnetite particles by the reaction of ferric chloride and iron powder in an alkaline medium. In many hydrothermal methods, a mixed aqueous solution of $\mathrm{Fe}^{2+}$ and $\mathrm{Fe}^{3+}$ is used as the starting solution and an oxidizing or reducing agent is added to the solution (Fan et al 2001; Wang et al 2004). This results in insufficient control of the properties because the reaction rate changes remarkably depending on the molar ratio of $\mathrm{Fe}^{2+} / \mathrm{Fe}^{3+}$ (Iida et al 2007) and the presence of oxidizing (Fan et al 2001) and reducing agents (Wang et al 2004). Therefore, it is necessary to clarify the reaction mechanism in order to control the properties although there have been no such studies so far.

In this paper, we have studied on the hydrothermal synthesis process without any oxidizing and reducing agents under various molar ratios of $\mathrm{Fe}^{2+} / \mathrm{Fe}^{3+}$. The particle size, crystallinity and magnetization properties of magnetite nanoparticles prepared were evaluated, and effects of the $\mathrm{Fe}^{2+} / \mathrm{Fe}^{3+}$ molar ratio on the reaction mechanism were analysed.

\section{Experimental}

All chemicals used in the experiments were of analytical reagent grade and were used without further purification. Ferrous sulfate $\left(\mathrm{FeSO}_{4}\right)$ and ferric chloride $\left(\mathrm{FeCl}_{3}\right)$ were 
dissolved in $40 \mathrm{ml}$ of deionized and deoxygenated water in a stainless steel autoclave. After several minutes, $20 \mathrm{ml}$ of $1 \mathrm{kmol} / \mathrm{m}^{3} \mathrm{NaOH}$ solution was added into the solution at a constant addition rate under vigorous stirring using a magnetic stirrer in an argon atmosphere. The addition rate of $\mathrm{NaOH}$ solution was set to be $3 \mathrm{ml} / \mathrm{min}$ for forming the precipitations uniformly. The initial $\mathrm{Fe}^{2+} / \mathrm{Fe}^{3+}$ molar ratio, $\alpha$, was varied in the range of $\alpha=0.25$ to 2 by adjusting the amount of $\mathrm{FeSO}_{4}$ under the constant amount of $\mathrm{FeCl}_{3}$ $(2 \mathrm{mmol})$. After air in the autoclave was replaced with argon, the sealed autoclave was maintained at $120^{\circ} \mathrm{C}$ for a given heating time in an electric oven and then cooled down to room temperature. The heating time was varied as 0 (i.e. without heating), 4 and $20 \mathrm{~h}$. The precipitate was washed with deionized water and decanted after centrifugation at $3000 \mathrm{rpm}$. After the washing operation was repeated 3 times, the sample was dried overnight in air at $80^{\circ} \mathrm{C}$.

The morphology of prepared samples was observed with a field emission scanning electron microscope (FE-SEM,
JEOL JSM-6700FW). The powder X-ray diffraction (XRD) patterns were measured with $\mathrm{CuK} \alpha$ radiation ranging from $2 \theta=10$ to $80^{\circ}$ at a scanning rate of $1 \%$ min using a Rigaku RINT-1500 powder X-ray diffractometer. The magnetic property was analysed using a superconducting quantum interference device (SQUID) magnetometer (Quantum Design model MPMS) at a magnetic field of $100 \mathrm{Oe}$ in the temperature range between 5 and $400 \mathrm{~K}$ for both field-cooling (FC) and zero-field-cooling (ZFC). The hysteretic cycles were recorded at room temperature in the range of magnetic field between -10000 and 10000 Oe.

\section{Results and discussion}

The reaction mechanism in the hydrothermal synthesis of $\mathrm{Fe}_{3} \mathrm{O}_{4}$ is as follows: ferrous hydroxide $\left(\mathrm{Fe}(\mathrm{OH})_{2}\right)$ grows on goethite $(\mathrm{FeOOH})$ which is transformed from ferric hydroxide $\left(\mathrm{Fe}(\mathrm{OH})_{3}\right)$, and then the $\mathrm{Fe}(\mathrm{OH})_{2}-\mathrm{FeOOH}$
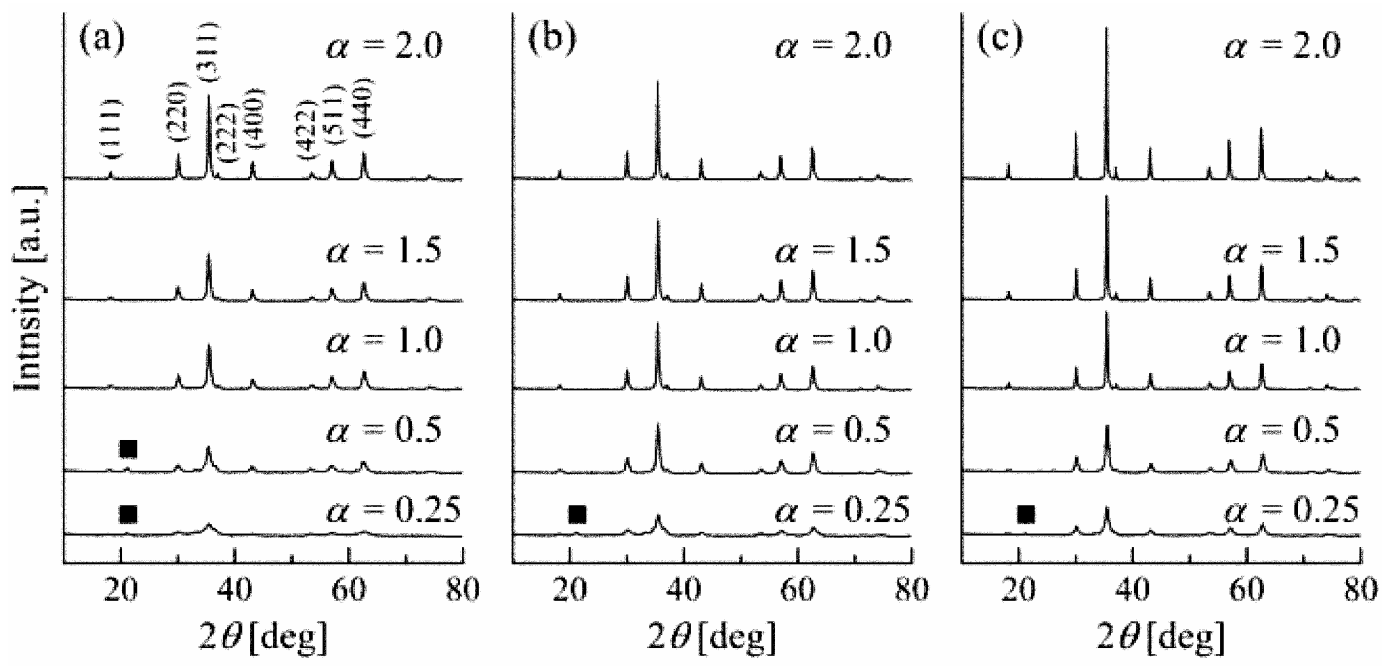

Figure 1. XRD patterns of samples prepared under various $\mathrm{Fe}^{2+} / \mathrm{Fe}^{3+}$ molar ratios, $\alpha$ (a) before heating and after heating for (b) $4 \mathrm{~h}$ and (c) $20 \mathrm{~h}$. The symbol a corresponds to the FeOOH diffraction line.
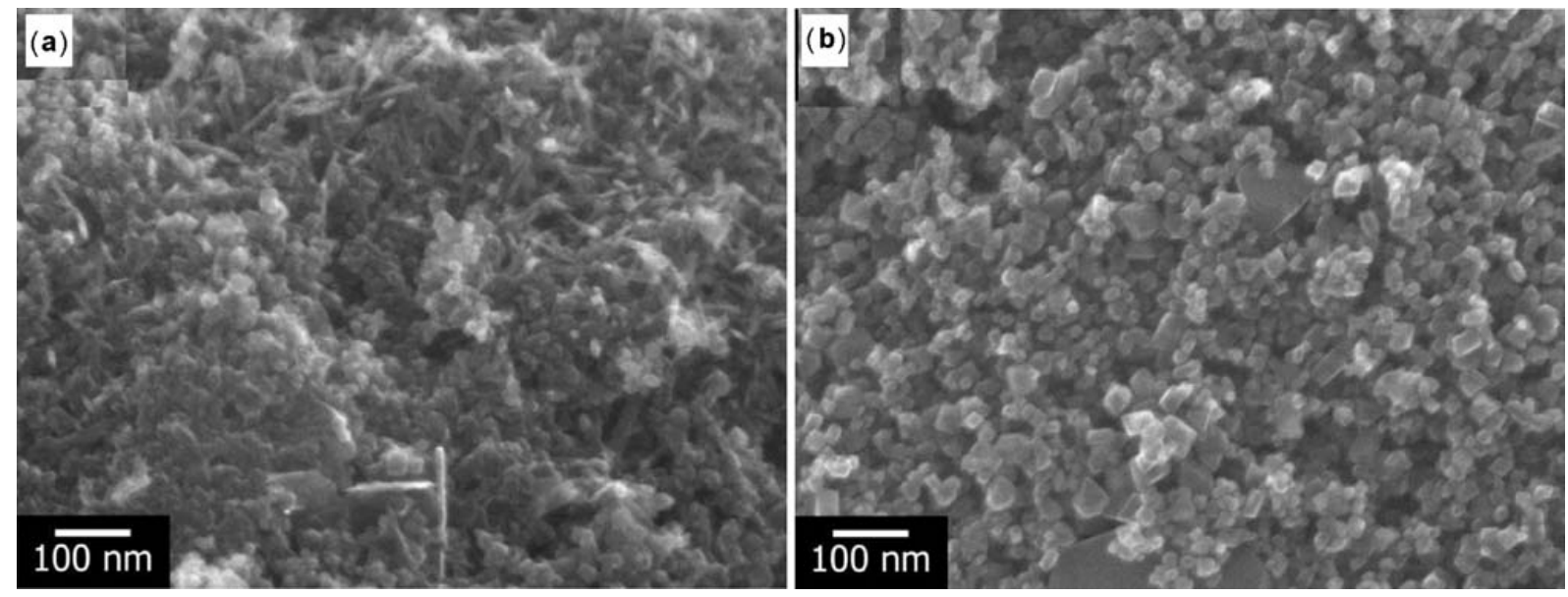

Figure 2. SEM images of samples obtained (a) before heating and (b) after heating for $20 \mathrm{~h}$ at $\alpha=0 \cdot 5$. 
composite is converted into homogeneous $\mathrm{Fe}_{3} \mathrm{O}_{4}$ according to the following reaction (Lian et al 2004)

$$
\mathrm{Fe}(\mathrm{OH})_{2}+2 \mathrm{FeOOH} \rightarrow \mathrm{Fe}_{3} \mathrm{O}_{4}+2 \mathrm{H}_{2} \mathrm{O} \text {. }
$$

Thus, the initial $\mathrm{Fe}^{2+} / \mathrm{Fe}^{3+}$ molar ratio of $\alpha=0.5$ is needed for the formation of $\mathrm{Fe}_{3} \mathrm{O}_{4}$. It is well known that this re-
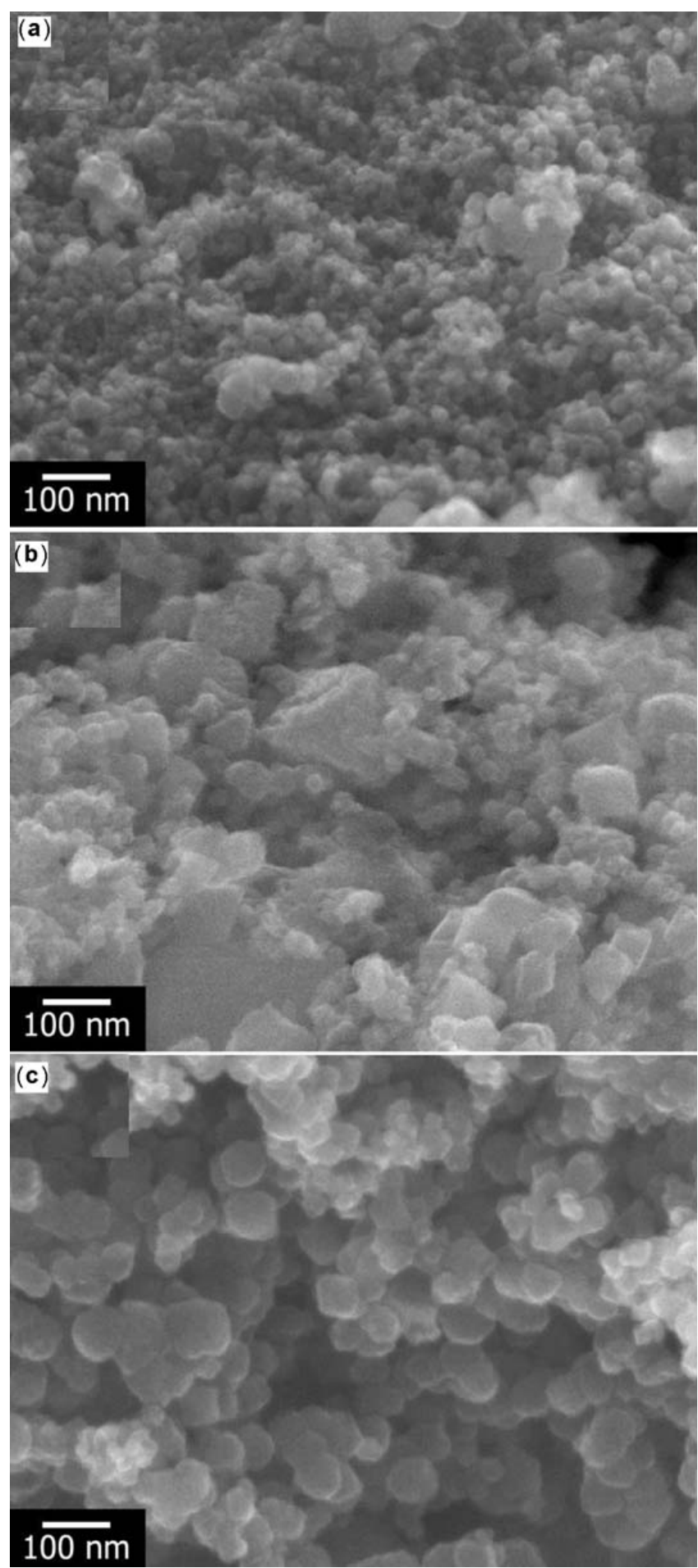

Figure 3. SEM images of samples obtained (a) before heating and after heating for (b) $4 \mathrm{~h}$ and (c) $20 \mathrm{~h}$ at $\alpha=2$. action is relatively slow at room temperature and that the reaction rate can be increased by heating. Figure 1 shows the XRD patterns of samples obtained after heating for a given time under various $\alpha$. Figures 2 and 3 indicate the SEM images of samples prepared under representative molar ratios of $\alpha=0.5$ and 2 as an example, respectively. In addition, the average crystallite size was calculated from the full-width at half-maximum (FWHM) of the $\mathrm{Fe}_{3} \mathrm{O}_{4}$ (311) diffraction peak at $2 \theta=35.5^{\circ}$ using Scherrer's equation. Figure 4 shows the change in the average crystallite size with the heating time. In the samples prepared at $\alpha \leq 0.5$ without heating, the typical peak of $\mathrm{FeOOH}$ phase was seen at $2 \theta \approx 21^{\circ}$ in the XRD patterns and the needle-shaped particles corresponding to $\mathrm{FeOOH}$ were observed as shown in figure 2(a) because of incomplete reaction. At $\alpha=0 \cdot 5, \mathrm{FeOOH}$ was consumed as the reaction progressed by heating and then homogeneous $\mathrm{Fe}_{3} \mathrm{O}_{4}$ phase with a relatively high crystallinity could be obtained. However, when $\alpha=0 \cdot 25$, FeOOH still remained after heating for $20 \mathrm{~h}$ because of the lack of $\mathrm{Fe}(\mathrm{OH})_{2}$. No peaks were observed in the XRD patterns at $2 \theta=14.9$, 23.7 and $26.0^{\circ}$, which are typical peaks of maghemite corresponding to (103) and (110) lines, (106) and (203) lines, and (213) and (116) lines, respectively. In addition, the lattice constant, $a$, calculated from the XRD patterns of the samples of $\alpha=0.5$ to 2 after heating for $20 \mathrm{~h}$ was between 8.37 and $8.42 \AA$, and close to that of magnetite $(8.396 \AA)$ compared to that of maghemite $(8.345 \AA)$. These also support that the samples of $\alpha \geq 0.5$ after heating were magnetite phase.

As can be seen in figure 2, at $\alpha=0.5$ the particle size after heating for $20 \mathrm{~h}$ was almost the same (about $30 \mathrm{~nm}$ ) as that before heating whereas the average crystallite size increased with increasing heating time as shown in figure 4. This implies that the reaction progresses rapidly by

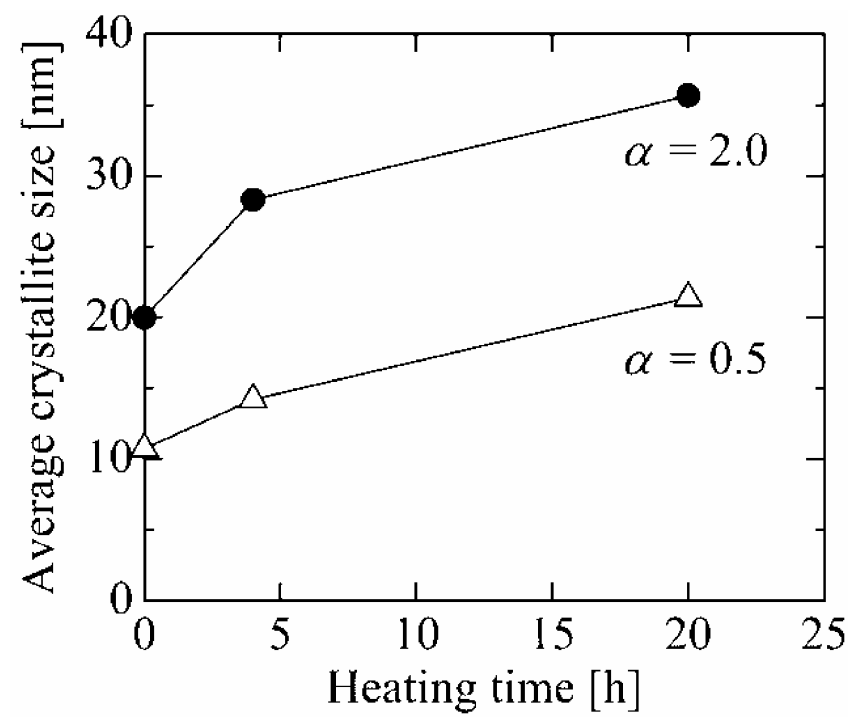

Figure 4. Change in average crystallite size of samples with heating time. 
heating and the crystal nucleation (i.e. formation of precursors) of $\mathrm{Fe}_{3} \mathrm{O}_{4}$ mainly occurs, resulting in inhibition of the crystal growth, and that the coalescence of crystallites in the inside of a particle increases the crystallinity.

On the other hand, when $\alpha \geq 1$ in which the amount of $\mathrm{Fe}(\mathrm{OH})_{2}$ in the starting solution exceeded the stoichiometric ratio, $\mathrm{FeOOH}$ was consumed almost completely before heating as shown in figures 1(a) and 3(a). Also, as can be seen in figures 2 and 3(a), the particle size before heating was almost the same as that at $\alpha=0 \cdot 5$. The results reveal that the reaction rate increases at room temperature in the presence of excess $\mathrm{Fe}(\mathrm{OH})_{2}$. In the heating period, the average crystallite size increased as the heating time elapsed similar to the case of $\alpha=0 \cdot 5$. However, the particle size and crystallinity increased greatly with increasing heating time. This tendency was remarkable as $\alpha$ increased, and especially at higher $\alpha$ for longer heating times,
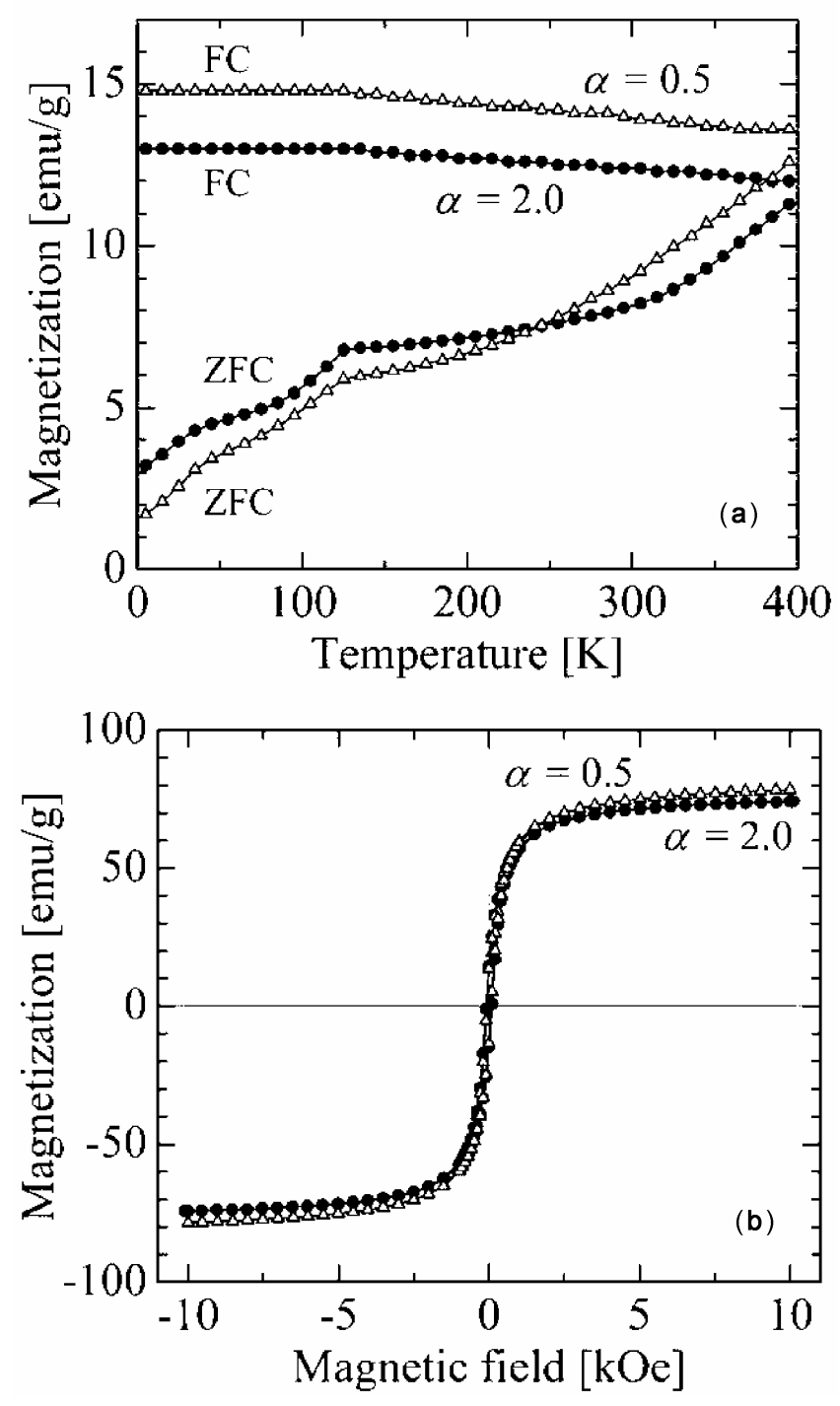

Figure 5. Magnetic properties of samples obtained after heating for $20 \mathrm{~h}$ : (a) magnetization-temperature curves and (b) magnetization-magnetic field curves. the nanoparticles with a shape close to a regular octahedron, which is the crystal form of $\mathrm{Fe}_{3} \mathrm{O}_{4}$ (Yu et al 2006), were obtained. The results suggest that the crystallization of $\mathrm{Fe}_{3} \mathrm{O}_{4}$ nanoparticles progresses in the heating period according to the different mechanisms depending on the initial $\mathrm{Fe}(\mathrm{OH})_{2}$ content; the growth of crystallites remarkably occurs at higher $\alpha$ in the heating period, caused by the Schikorr reaction in which $\mathrm{Fe}(\mathrm{OH})_{2}$ is transformed into $\mathrm{Fe}_{3} \mathrm{O}_{4}$ at higher temperatures according to the following reaction (Schikorr 1929; Shipko and Douglas 1956)

$$
3 \mathrm{Fe}(\mathrm{OH})_{2} \rightarrow \mathrm{Fe}_{3} \mathrm{O}_{4}+\mathrm{H}_{2}+\mathrm{H}_{2} \mathrm{O} .
$$

The rate of the Schikorr reaction is very slow as compared with that of the reaction expressed by (1). Therefore, in the presence of excess $\mathrm{Fe}(\mathrm{OH})_{2}$ the crystal nucleation was completed rapidly at room temperature according to (1), and then using surplus $\mathrm{Fe}(\mathrm{OH})_{2}$ the crystallites in the $\mathrm{Fe}_{3} \mathrm{O}_{4}$ nanoparticles grew up slowly under hydrothermal conditions according to (2) rather than their coalescence.

Figure 5 shows the magnetization-temperature (i.e. $\mathrm{ZFC} / \mathrm{FC}$ ) curves and the magnetization-magnetic field hysteresis of samples obtained after heating for $20 \mathrm{~h}$. The Verwey transition and hysteresis curves were observed in both samples. The $\mathrm{Fe}_{3} \mathrm{O}_{4}$ nanoparticles prepared in this work exhibited a ferromagnetic behaviour with a high saturation magnetization of about $80 \mathrm{emu} / \mathrm{g}$, which was higher than that of maghemite $(56 \mathrm{emu} / \mathrm{g})$ and, however, somewhat lower than that of the corresponding bulk $(92 \mathrm{emu} / \mathrm{g}$ ) due to their smaller size (Lee et al 1996); this result was similar to those reported by Zaitsev et al (1999) and Yu et al (2006). Accordingly, it has been clarified that the $\mathrm{Fe}_{3} \mathrm{O}_{4}$ nanoparticles consist of almost the same magnetic phase (Maity and Agrawal 2007) and have similar magnetite properties regardless of $\alpha$ whereas the particle size and crystallinity change depending on $\alpha$.

\section{Conclusions}

The hydrothermal synthesis of $\mathrm{Fe}_{3} \mathrm{O}_{4}$ nanoparticles were carried out without adding any oxidizing and reducing agents under various initial $\mathrm{Fe}^{2+} / \mathrm{Fe}^{3+}$ molar ratios in order to clarify effects of the molar ratio on the reaction mechanism. When the molar ratio was 0.5 corresponding to the stoichiometric ratio in the synthesis reaction of $\mathrm{Fe}_{3} \mathrm{O}_{4}$ from $\mathrm{Fe}(\mathrm{OH})_{2}$ and $\mathrm{FeOOH}$, the nucleation of $\mathrm{Fe}_{3} \mathrm{O}_{4}$ crystals progressed rapidly in an initial stage of the hydrothermal synthesis, resulting in formation of the $\mathrm{Fe}_{3} \mathrm{O}_{4}$ nanoparticles having a smaller size and a lower crystallinity. On the other hand, when the molar ratio was more than 1, the particle size and crystallinity increased with increasing molar ratio because the crystallites of $\mathrm{Fe}_{3} \mathrm{O}_{4}$ nanoparticles grew up slowly using surplus $\mathrm{Fe}(\mathrm{OH})_{2}$ under hydrothermal conditions according to the Schikorr reaction. The $\mathrm{Fe}_{3} \mathrm{O}_{4}$ nanoparticles prepared under various 
molar ratios had good magnetic properties regardless of the molar ratio. The results suggest that the molar ratio is one of the most important operating factors in the control of characteristics of $\mathrm{Fe}_{3} \mathrm{O}_{4}$ nanoparticles.

\section{References}

Fan R, Chen X H, Gui Z, Liu L and Chen Z Y 2001 Mater. Res. Bull. 36497

Gotić M, Jurkin T and Musić S 2007 Colloid Polym. Sci. 285 793

Iida H, Takayanagi K, Nakanishi T and Osaka T $2007 \mathrm{~J}$. Colloid Interf. Sci. $\mathbf{3 1 4} 274$

Lee J, Isobe T and Senna M 1996 Colloids Surf. A109 121

Lian S, Wang E, Kang Z, Bai Y, Gao L, Jiang M, Hu C and Xu L 2004 Solid State Commun. 129485
Maity D and Agrawal D C 2007 J. Magn. Magn. Mater. 308 46

Park J et al 2004 Nat. Mater. 3891

Qian Y, Xie Y, He C, Li J and Chen Z 1994 Mater. Res. Bull. 29953

Schikorr G 1929 Z. Elektrochemie 3565

Shipko F J and Douglas D L 1956 J. Phys. Chem. 601519

Wang J, Peng Z, Huang Y and Chen Q 2004 J. Cryst. Growth 263616

Wang X, Zhang C, Wang X and Gu H 2007 Appl. Surf. Sci. 253 7516

Yu W, Zhang T, Zhang J, Qiao X, Yang L and Liu Y 2006 Mater. Lett. 602998

Zaitsev V S, Filimonov D S, Presnyakov I A, Gambino R J and Chu B 1999 J. Colloid Interf. Sci. 21249

Zhou Z H, Wang J, Liu X and Chan H S O 2001 J. Mater. Chem. 111704 